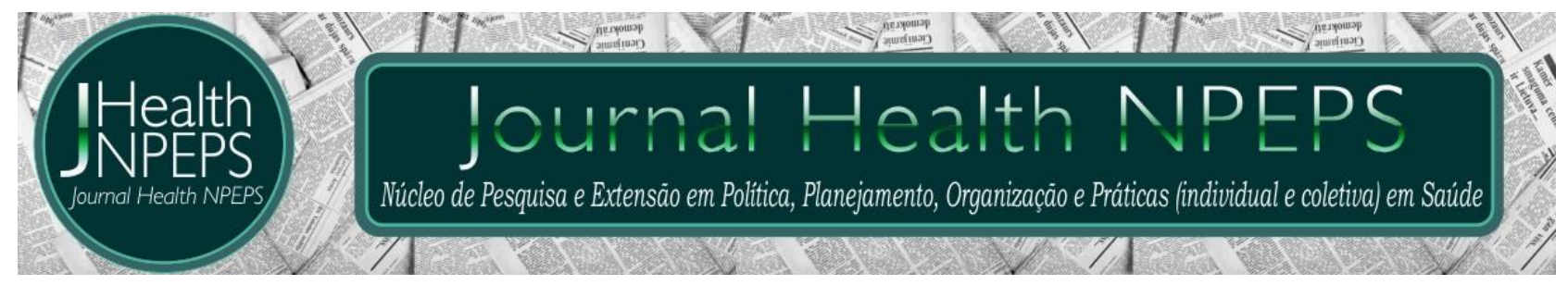

http://dx.doi.org/10.30681/252610105017

ARTIGO ORIGINAL

\title{
Características resilientes y consumo de alcohol en adolescentes que viven en situación vulnerable
}

\section{Resilient characteristics and alcohol consumption in adolescents living in a vulnerable situation}

\section{Características de resiliência e consumo de álcool em adolescentes em situação de vulnerabilidade}

\section{Fani Villa-Rivas ${ }^{1}$, Rocio Castillo-Díaz ${ }^{2}$, Nadia Velázquez-Hernández ${ }^{3}$, José David Montelongo-Lugo ${ }^{4}$}

\section{RESUMEN}

Objetivo: determinar la relación entre el uso de alcohol y las características resilientes en adolescentes que viven en situación vulnerable. Método: diseño descriptivocorrelacional y transversal. Muestra de 234 adolescentes de secundaria del área vulnerable de Durango, México. Se utilizó una cédula de datos personales para la información sociodemográfica de los participantes, al igual que la aplicación de AUDIT y el inventario de características personales de resiliencia. Resultados: el 53.0\% fueron hombres, 54.7\% entre 13 a 14 años, $75.2 \%$ únicamente estudia, 54.7\% su familia es con padre y madre. El $15 \%$ mostró consumo sensato, el 5.6\% uso dependiente y $13.2 \%$ uso dañino. Los participantes de 15 a 16 años mostraron mayor uso de alcohol $(p<.05)$, el $70.8 \%$ (IC 95\% [51-90]) han consumido alcohol alguna vez en la vida, 50\% (IC 95\% [28-72]) en el último año, 41.7\% (IC 95\% [20-63]) en el último mes. Las subescalas Autoestima $\left(r_{s}=-1.59, p<.05\right)$, Humor $\left(r_{s}=-1.29, p<.05\right)$ y Creatividad $\left(r_{s}=-1.43, p<.05\right)$ y uso de alcohol se correlacionaron negativamente. Conclusión: participantes entre 15 y 16 años mostraron mayor uso de alcohol y del inventario de características personales de resiliencia: el autoestima, humor y creatividad mostraron relación con el uso de alcohol.

Descriptores: Resiliencia; Consumo de Alcohol en Menores; Adolescentes.

\footnotetext{
${ }^{1}$ Enfermera. Doctora en Salud Pública. Profesora de tiempo completo. Líder del Cuerpo Académico Cuidado Social de la Salud. Universidad Juárez del Estado de Durango. Durango, México. E-mail: fani@ujed.mx ORCID ID: http://orcid.org/0000-0001-7222$\underline{5151}$

${ }^{2}$ Enfermera. Doctora en Ciencias de la Educación. Profesora de tiempo completo. Universidad Juárez del Estado de Durango. Durango, México. E-mail: rocio.castillo@ujed.mx ORCID ID: http://orcid.org/0000-0002-4789-828X Autor correspondiente: Blv. Juan Pablo II, núm. 512, Col. Ruiz Masie, Durango, México.

${ }_{3}^{3}$ Médica. Doctora en Ciencias Médicas. Profesor-Investigador de tiempo completo. Universidad Juárez del Estado de Durango. Durango. México E-mail: nadia.velazquez@ujed.mx ORCID ID: http://orcid.org/0000-0002-7221-6535

${ }^{4}$ Enfermero. Licenciado en Enfermería. Universidad Juárez del Estado de Durango. Durango, México. E-mail: neptunodgo21@gmail.com ORCID ID: https://orcid.org/0000-0002-0976-7086
} 


\section{ABSTRACT}

Objective: to determine the relationship between alcohol use and resilient characteristics in adolescents living in a vulnerable situation. Method: descriptivecorrelational cross design. Sample of 234 high school adolescents from the vulnerable area of Durango, Mexico. A Personal Data Card was used for the sociodemographic information of the participants, as well as the application of the AUDIT and the Inventory of Personal Resilience Characteristics. Results. 53.0\% were men, with 54.7\% between 13 and 14 years old, 75.2\% studied only, 54.7\% had a family with father and mother. 15\% had sensitive consumption, 5.6\% dependent use and 13.2\% harmful use. Participants aged 15 to 16 years had a higher use of alcohol ( $p<0.05), 70.8 \%(95 \% \mathrm{Cl}$ [51-90]) consumed alcohol at some time in their lives, 50\% (95\% Cl [28-72] ]) in the last year, and $41.7 \%(95 \% \mathrm{Cl}$ [20-63]) in the last month. The subscales Self-esteem ( $r$ s = 1.59, $p<0.05)$, Humor ( $r s=-1.29, p<0.05)$ and Creativity $(r s=-1.43, p<0.05)$ and alcohol use were negatively correlated. Conclusion: participants between 15 and 16 years old showed greater use of alcohol and the inventory of personal characteristics of resilience: self-esteem, humor and creativity showed a relationship with the use of alcohol.

Descriptors: Resilience; Underage Drinking; Adolescents.

\section{RESUMO}

Objetivo: verificar a relação entre uso de álcool e características de resiliência em adolescentes em situação de vulnerabilidade. Método: desenho descritivo-correlacional transversal. Amostra de 234 adolescentes do ensino médio da área vulnerável de Durango, México. Foi utilizado um formulario de dados pessoais para as informações sociodemográficas dos participantes, bem como a aplicação do AUDIT e do Inventário de Características de Resiliência Pessoal. Resultados: 53,0\% eram homens, sendo 54,7\% entre 13 e 14 anos, 75,2\% estudavam apenas, 54,7\% a família era com pai e mãe. $15 \%$ apresentaram consumo sensível, $5,6 \%$ uso dependente e $13,2 \%$ uso prejudicial. Participantes de 15 a 16 anos apresentaram maior uso de álcool ( $p<0,05), 70,8 \%$ (IC 95\% [51-90]) consumiram álcool alguma vez na vida, 50\% (IC 95\% [28-72]) no último ano, 41,7\% (IC 95\% [20-63]) no último mês. As subescalas Autoestima ( $r s=-1,59, p<0,05$ ), Humor ( $r s=-1,29, p<0,05)$ e Criatividade ( $r s=-1,43, p<0,05)$ e uso de álcool foram negativamente correlacionadas. Conclusão: participantes entre 15 e 16 anos apresentaram maior uso de álcool e o inventário de características pessoais de resiliência: autoestima, humor e criatividade mostrou relação com o uso de álcool.

Descritores: Resiliência; Consumo de Alcool em Menores; Adolescentes.

\section{INTRODUCCIÓN}

El consumo de alcohol constituye un reto para la salud pública en todo el mundo, debido a las nocivas repercusiones económicas y sociales que en términos de productividad laboral y escolar ocasiona. Los adolescentes presentan mayor vulnerabilidad ante este fenómeno por una serie de cambios físicos, emocionales, sociales, culturales, en su rol familiar, y en la creciente adquisición de la autonomía, problemas cognitivos ${ }^{1,2}$. Lo anterior demanda una atención particular en el área de la salud, ya que el consumo de 
alcohol y otras drogas son causantes principales de lesiones, accidentes de tráfico, violencia y muertes prematuras. Además pueden provocar problemas de salud en etapas posteriores de la vida ${ }^{3,4}$.

La OMS dio a conocer en el Informe de la situación mundial sobre el alcohol y Salud 2018, que 155 millones de adolescentes en edades entre 15 a 19 años son bebedores de alcohol a nivel mundial, presentando a menudo un consumo excesivo de alcohol, y en el año 2016 el consumo excesivo de alcohol causó 3.3 millones de muertes a nivel mundial $^{5}$. El alcohol es considerado como una droga legal, cuyos efectos son depresores del sistema nervioso central. Su consumo en dosis mayores produce embriaguez caracterizada por alteraciones en el comportamiento, el razonamiento y la capacidad perceptiva. Si la depresión del sistema nervioso es intensa, puede llegar a generar coma etílico ${ }^{6}$. La Comisión Nacional para el Desarrollo y Vida sin Drogas, afirma que el alcohol sigue siendo la droga más consumida por los adolescentes escolares, con una edad de incio de consumo aproximadamente a los 13 años, se estima que uno de cada cuatro adolescentes, es decir el 25\% afirma haber consumido alcohol alguna vez en la vida ${ }^{7}$.
La adolescencia es considerada como una etapa critica en la vida, donde se generan grandes cambios fisicos y mentales, el adolescente desarrolla su propia identidad, intereses, y habilidades necesarias para desarrollarse en la vida adulta. También es una etapa de riesgos por su natural deseo de experimentación, pueden estar expuestos $a$ situaciones violentas, discriminación y violencia de género, embarazos y consumo de alcohol y otras drogas $^{8}$.

Por otra parte, la resiliencia es la capacidad de mantener la integridad luego de atravesar un momento que los modifica al pasar por situaciones adversas que se les presenta en el transcurso de su desarrollo y crecimiento $^{9}$. La resiliencia es definida como la capacidad de los seres humanos sometidos a los efectos de una adversidad, de superarla e incluso salir fortalecidos de la situación, por ello resiliencia se teje, no hay que buscarla sólo en la interioridad de la persona ni en su entorno, porque anuda constantemente un proceso íntimo con el entorno social ${ }^{10}$.

Por lo anteriormente expuesto se formuló el objetivo del presente estudio de investigación fue determinar la relación entre el uso de alcohol y las 
características

resilientes

en adolescentes que viven en situación vulnerable.

\section{MÉTODO}

El diseño del estudio fue descriptivo, correlacional y transversal $^{11}$. El estudio se llevó a cabo durante el mes de agosto de 2019, La muestra tipo censo estuvo conformada por el total de estudiantes que fueron 234 adolescentes que viven en situación vulnerable, considerando la zona geográfica de la escuela y el lugar donde viven como zona de marginación, violencia, con antecedentes de presentar riesgos como el consumo de drogas y embarazos a temprana edad, así como también bajo nivel académico de sus padres ${ }^{12}$ y que estudian en una escuela secundaria del área urbana de la ciudad de Durango, México. Para la selección de los participantes se considero que participaran el $100 \%$ de los estudiantes inscritos en la secundaria pública.

Para la recolección de datos se utilizó una cédula de datos personales, integrada por cinco reactivos que preguntaron género, edad, grado escolar, ocupación y tipo de familia. Para medir el consumo de alcohol se aplicó una cédula de consumo de alcohol, la cual indago sobre la edad de inicio de consumo, las prevalencias, frecuencia y cantidad compuesta de 10 reactivos.

El siguiente instrumento fue el cuestionario de identificación de trastornos debidos al consumo de alcohol, Alcohol Use Disorder Identificacionn Test (AUDIT) consta de 10 preguntas y el inventario para evaluar la presencia de cinco factores personales de resiliencia que consta de 48 ítems, la OMS ha recomendado su uso en diversos países $^{13}$.

El instrumento AUDIT fue desarrollado como parte de un estudio colaborativo de deteccionn y manejo de los problemas relacionados al alcohol en atencionn primaria, en seis pai口ses (Australia, Bulgaria, Kenya, Merxico, Noruega y Estados Unidos) y bajo la tutela de la OMS, que ha recomendado su uso y validacionn en diversos pai口ses. El AUDIT es un instrumento de tamizaje cuyos reactivos examinan el consumo excesivo de alcohol y permiten identificar a individuos con patrones de consumo de riesgo o perjudicial de dependencia al consumo de alcohol 59. Consta de 10 preguntas, las 8 primeras hacen referencia a los unltimos 12 meses y las 2 unltimas a toda la vida, dividido 
en tres dominios, que consideran por separado: dominio 1, consumo de alcohol (iatems 1 al 3), dominio 2, siantomas de dependencia (iatems 4 al 6) y dominio 3, consecuencias negativas del consumo (jatems 7 al 10). Las preguntas 1 a la 8 punturan de 0 a 4 y las preguntas 9 y 10 punturan 0, 2 o 4 donde el puntaje marximo es de 40 puntos En el presente estudio mostro un Alpha de Cronbach de 84.

Inventario de Factores Personales de Resiliencia, creado por Salgado (2004) ${ }^{13}$. El inventario comprende 48 items dirigidos a evaluar la presencia de cinco factores personales de resiliencia, redactados tanto en forma negativa como positiva, cuya modalidad de respuesta es sí y no. Autoestima Ítems Positivos 1,2, $11,12,20,21$, Ítems negativos 30, 31, 40, 41 (10 - 9 Alta, 8 -6 Medio, 5 - 1 Bajo) Empatía Ítems Positivos 4, 13, 22, 23, 42 Ítems negativos 3, 32, 33, 43, 44 (10 - 8 Alto, 7 - 5 Medio, 4 -1 Bajo), Autonomía Ítems Positivos 5, 6, 14, 15, 24, 25 Ítems negativos 34, 35, 45, 46 (10 - 8 Alto, 7 4 Medio, 3 - 1 Bajo), Humor Ítems Positivos 7, 8, 16, 17, 26 Ítems negativos 27, 36, 37, 47, 48 (10 - 7 Alto, 6 - 4 Medio, 3 - 1 Bajo) y Creatividad Ítems Positivos 9, 10, 18, 19, 28 Ítems negativos 29, 38, 39 (8-6 Alto, 5 -3 Medio, 2 - 1 Bajo).

El presente estudio de investigación contó con la aprobación del Cuerpo Académico Cuidado Social de la Salud, así como de los Directivos de la Secundaria donde fue realizado el estudio. Se contó con el Consentimiento Informado de los padres de famiia o tutores de los adolescentes y el asentimiento de los participantes. Se brindo información clara respecto al objetivo del estudio, se dieron a conocer los instrumentos, antes de iniciar con la aplicación de los instrumentos del estudio.

El total de los estudiantes aceptaron participar en el estudio, previa confirmación de fecha. Se les aseguro conservar la privacidad y anonimato de los datos proporcionados. La recolección de datos se llevó a cabo en el mismo período, en cada salón de clases alejados de ruido y con apoyo de encuestadores con el fin de que sus respuestas fueran lo más verdaderas posibles.

Primero se aplicó la cedula de datos personales, después se aplicó la cedula de consumo de alcohol, finalmente para concluir se aplicó la escala de características resilientes. Al término, las encuestas se colocaron en 
una caja forrada para dar más seguridad a los estudiantes de que la información era confidencial. Los instrumentos quedaron bajo resguardo por los autores del estudio por seis meses $y$ posteriormente se destruirán.

En la escuela secundaria donde se aplicarón las encuestas, para retribuir la autorización otorgada para la aplicación de los instrumentos se realizó una Feria Integral de la salud, platicas y talleres, Proyecto de Vida Proyecto líder, impartidos por los autores del estudio y otras organizaciones, Instituto Estatal de la Juventud y Centros de Integración Juvenil. El estudio se apegó a lo establecido en el Reglamento de la Ley General de Salud en Materia de Investigación para la Salud ${ }^{15}$.

\section{RESULTADOS}

De acuerdo a los resultados obtenidos, predominó el género masculino con un $53.0 \%$ el $54.7 \%$ de los participantes se situaron en el rango de edad de los 13 a 14 años, con respecto a la ocupación el $75.2 \%$ se dedica únicamente a estudiar, y en cuanto a la estructura familiar el $54.7 \%$ se encuentran integrados en una familia completa.

Tabla 1 - Consistencia interna de los instrumentos. $(n=234)$

\begin{tabular}{|c|c|c|c|}
\hline Instrumento & Reactivos & $\begin{array}{l}n . \\
\text { reactivos }\end{array}$ & $\begin{array}{l}\text { Alpha de } \\
\text { Cronbach }\end{array}$ \\
\hline Inventario & $1-48$ & 48 & .77 \\
\hline S.E. Autoestima & $\begin{array}{c}1,2,11,12,20,21,30 N, 31 N, \\
40 N, 41 N\end{array}$ & 10 & .70 \\
\hline S.E. Empatía & $\begin{array}{c}4,13,22,23,42,3 N, 32 N \\
33 N, 43 N, 44 N\end{array}$ & 10 & .65 \\
\hline S.E. Autonomía & $\begin{array}{c}5,6,14,15,24,25,34 N, 35 N, \\
45 N, 46 N\end{array}$ & 10 & .60 \\
\hline S.E. Humor & $\begin{array}{c}7,8,16,17,26,27 N, 36 N, 37, \\
47 N, 48 N\end{array}$ & 10 & .64 \\
\hline S.E. Creatividad & $\begin{array}{c}9,10,18,19,28,29 N, 38 N, \\
39 N\end{array}$ & 8 & .60 \\
\hline APGAR & $1-5$ & 5 & .72 \\
\hline AUDIT & $1-10$ & 10 & .91 \\
\hline
\end{tabular}

Nota: $A P G A R=$ Escala de evaluación Familiar. $A U D I T=$ Cuestionario de Identificacion de los Trastornos Debidos al Consumo de Alcohol, S.E= Subescalas.

Tabla 2- Prevalencia global, lápsica, actual e instantánea del consumo de alcohol. $(\mathbf{n = 2 3 4 )}$

\begin{tabular}{|c|c|c|c|c|}
\hline \multirow[t]{2}{*}{ Prevalencias de consumo } & \multirow{2}{*}{$f$} & \multirow{2}{*}{$\%$} & \multicolumn{2}{|c|}{ IC 95\% } \\
\hline & & & $\mathrm{LI}$ & $L S$ \\
\hline Alguna vez en la vida & 79 & 33.8 & 28 & 40 \\
\hline En el último año & 50 & 21.4 & 16 & 27 \\
\hline En el último mes & 33 & 14.1 & 10 & 19 \\
\hline En la última semana & 23 & 9.8 & 6 & 14 \\
\hline
\end{tabular}

Nota: IC= Intervalo de Confianza, LI= Límite Inferior, LS= Limite Superior. 
La consistencia interna de los instrumentos utilizados en el estudio para medir la Resiliencia reportó un Alpha de Cronbach de .77 para la escala general. En las dimensiones del inventario se presentó un Alpha de Cronbach que osciló entre $\begin{array}{llll}.60 & a & .70\end{array}$ .Cuestionario de Identificacion de los Trastornos Debidos al Consumo de Alcohol (AUDIT) Alpha de Cronbach de .72. Las subescalas de Resiliencia presentaron una consistencia interna de .60. Escala de evaluación Familiar .72.

La Tabla 2 muestra las prevalencias de consumo de alcohol. El $33.8 \%$ (IC95\% [28-40]) de los participantes consumieron alcohol alguna vez en la vida, el 21.4\% (IC95\% [16-27]) en el último año, el $14.1 \%$ (IC95\% [10-19]) en el último mes y el 9.8\% (IC95\% [6-14]) en la última semana.

Respecto a las prevalencias de consumo de alcohol por género, fueron mayores en los hombres que en las mujeres, sin embargo no se encontraron diferencias estadísticamente significativas.

En lo que se refiere a las prevalencias de consumo de alcohol por rangos de edad, se registraron prevalencias mayores en los participantes de rango de edad de 15 a
16 años, presentando diferencias estadísticamente significativas $(p<.05)$, en las prevalencias de consumo alguna vez en la vida con $70.8 \%$ (IC 95\% [5190]), en el último año con 50\% (IC 95\% [28-72]), en el último mes con 41.7\% (IC 95\% [20-63]) y en la útima semana con $33.3 \%$ (IC 95\% [13-54]).

La tabla 3 muestra los tipos de consumo de alcohol según el AUDIT. El $15 \%$ de los participantes que consumen alcohol mostraron un Consumo Sensato, el $5.6 \%$ un Consumo Dependiente y el 13.2\% un Consumo Dañino.

La tabla 4 muestra que el índice de la Escala General de la Resiliencia $\left(r_{s}=-1.62, \quad p<.01\right)$, además de las subescalas de Autoestima ( $r_{s}=-1.59$, $p<.05)$, Humor $\left(r_{s}=-1.29, \quad p<.05\right)$ y Creatividad $\quad\left(r_{s}=-1.43, \quad p<.05\right)$ se correlacionaron de manera negativa y significativa con el índice del AUDIT, lo que indica que a mayor nivel de resiliencia y de los componentes de la resiliencia como lo son la Autoestima, Humor y Creatividad, es menor del consumo riesgoso de alcohol. 
Tabla 3 - Frecuencias de consumo de alcohol según AUDIT.

\begin{tabular}{lcc}
\hline Consumo de Alcohol & $F$ & $\%$ \\
\hline Sensato & 35 & 15.0 \\
Dependiente & 13 & 5.6 \\
Dañino & 31 & 13.2 \\
\hline
\end{tabular}

Nota: $f=$ frecuencia $\%=$ porcentaje, $I C=$ Intervalo de Confianza.

Tabla 4 - Coeficiente de Correlación de Spearman para la resiliencia y subescalas de la resiliencia

\begin{tabular}{lcccccc}
\hline Variable & $\begin{array}{c}\text { Indice Escala } \\
\text { General de } \\
\text { Resiliencia }\end{array}$ & $\begin{array}{c}\text { Índice } \\
\text { Autoestima }\end{array}$ & $\begin{array}{c}\text { Índice } \\
\text { Empatía }\end{array}$ & $\begin{array}{c}\text { Índice } \\
\text { Autonomía }\end{array}$ & $\begin{array}{c}\text { Índice } \\
\text { Humor }\end{array}$ & $\begin{array}{c}\text { Índice } \\
\text { Creatividad }\end{array}$ \\
\hline $\begin{array}{l}\text { Indice } \\
\text { AUDIT }\end{array}$ & $-.162^{*}$ & $-.159^{*}$ & .042 & -.083 & $-.129^{*}$ & $-.143^{*}$ \\
\hline
\end{tabular}

Nota: ${ }^{* *}=p<.01,{ }^{*}=p<.05 ;$ valores bilaterales

\section{DISCUSIÓN}

Respecto a las características sociodemográficas de la muestra, fue mayor la cantidad de hombres participantes, predominó el rango de edad entre 13 y 14 años, con respecto a la ocupación la mayoría se dedica únicamente a estudiar y pertenece a una familia completa, estos resultados concuerdan con investigaciones previas ${ }^{16}$ 18.

Las prevalencias del consumo de alcohol fueron mayores en los participantes de 15 a 16 años, presentando diferencias estadísticamente significativas en las prevalencias de consumo alguna vez en la vida, en cuanto al tipo de consumo el $5.6 \%$ mostró consumo sensato, el $13.2 \%$ consumo dependiente y el $13.2 \%$ consumo dañino, estas prevalencias son mas bajas que las reportadas en estudios anteriores ${ }^{19,20 .}$

Respecto a las prevalencias del consumo de alcohol fueron mayores en los hombres que en las mujeres, sin embargo no se encontraron diferencias estadísticamente significativas. El género masculino puede jugar un papel determinante para el inicio del consumo de estas sustancias en los adolescentes, por lo tanto es un factor importante que debe ser considerado en próximas investigaciones que estén centradas en el diseño de intervenciones en adolescentes ${ }^{21}$. Estos resultados muestran que el consumo de alcohol en los adolescentes presenta un problema prioritario de salud, lo cual hace necesario el diseño e implementación de programas de prevención dirigidas a este grupo de la sociedad. 
Acerca de la Escala General de la Resiliencia se correlacionaron de manera negativa y significativa con el índice AUDIT, lo que indica que a mayor nivel de resiliencia y de los componentes de la resiliencia como lo son la Autoestima, Humor y Creatividad, es menor el consumo riesgoso de alcohol, resultados similares encontraron Alonso y colaboradores ${ }^{15}$ además de otros autores con investigaciones previas $22-24$ con las subescalas de Resiliencia; competencia personal y aceptación de sí mismo mostrando relación significativa inversa con el consumo de alcohol, indicando que a mayor puntaje en la Escala de Resiliencia menor es el consumo de alcohol ${ }^{24}$.

Lo anterior deja en evidencia que al fortalecer las características resilientes se le permite al individuo enfrentar y resistir la presión social de los pares o la tentación de experimentar haciendo uso de estrategias de control y manejo asertivas, evitando el consumo de sustancias adictivas por lo tanto la resiliencia se convierte en un determinante factor protector.

El presente estudio de investigación presenta la limitación que se realizó en una sola institución educativa de nivel secundaria.

\section{CONCLUSIÓN}

En base a los resultados obtenidos en el presente estudio de investigación, se encontró que los participantes del rango de edad de 15 y 16 años mostraron mayor consumo de alcohol, del inventario de características personales de resiliencia: autoestima, humor y creatividad mostraron relación con el consumo de alcohol. Este hallazgo de investigación es relevante para en un futuro diseñar $e$ implementar intervenciones para la prevención del consumo de alcohol.

\section{REFERENCIAS}

1. Brown SA, McGue M, Maggs J, Schulenberg J, Hingson R, Swartzwelder $S$, et al. A developmental perspective on alcohol and youths 16 to 20 years of age. Pediatrics. 2008; 121(Suppl 4):290310.

2. Windle M, Spear L, Fuligni A, Angolld A, Brown J, Pine D, et al. Transitions into underage and problem drinking: developmental processes and mechanisms between 10 and 15 years of age. Pediatrics. 2008; 121(Suppl 4):273-289. 
3. Organización de Mundial de la Salud [OMS]. Adolescentes: riesgos para la salud y soluciones. Nota descriptiva. Centro de prensa; 2017.

4. Martínez-Rodríguez MR, CoronadoHernández MJ, Betancourt-Esparza MC, Díaz-Oviedo AD, GallegosMartínez J. Drogas lícitas e ilícitas: consumo de los estudiantes en una Facultad de Enfermería. Enf Neurol Méx. 2010; (10):130-134.

5. Organización Mundial de la Salud. Informe de situación mundial sobre el alcohol y la salud 2018. Organización Mundial de la Salud. Disponible en: http: / /apps.who.int/iris/handle/1066 5/274603.

6. World Health Organization. Global status report on alcohol and health. 2014. Available from: https://apps.who.int/iris/bitstream/h andle/10665/112736/9789240692763_ eng.pdf;jsessionid=56EFE9111DE50A6D CD9C864C6D1C927D?sequence $=$.

7. Organización Mundial de la Salud. La prueba de detección de consumo de alcohol, tabaco y sustancias (ASSIST), Manual para uso en la atención primaria. Organización Mundial de la Salud.2010 Disponible en: https://www.who.int/substance_abus e/activities/assist_screening_spanish. pdf
8. UNICEF México. Los derechos de la infancia y la adolescencia en México. Disponible en: https://www.unicef.org/mexico/medi a/1791/file/SITAN-UNICEF.pdf

9. Vanistendael S. La reqsilience ou le reqalisme de l'esperance: blesse ${ }^{\circ}$ mais pas vaincu. Ginebra: Bureau International Catholique de l'Enfance; 1996.

10. Cyrulnik Boris. La maravilla del dolor, el sentido de la resiliencia. Barcelona; 2001.

11. Burns N, Grove SK. Investigación en Enfermería. Barcelona: Manual Moderno; 2016.

12. CEPAL. Informe de la Reunión de Expertos: Seminario Internacional sobre las Diferentes Expresiones de la Vulnerabiliad Social en América Latina y el Caribe, División de Población de la Cepal/Celade, Santiago de Chile, Chile; 2001.

13. Organización Mundial de la Salud (OMS) Cuestionario de identificación de los trastornos debidos al consumo de alcohol. 1982 Disponible en: http://www.who.int/substance_abu se/activities/en/AUDITmanualSpanis h.pdf?ua $=1$

14. Salgado C. Métodos e instrumentos para medir la resiliencia. Una 
alternativa peruana. Liberabit. 2005; (11):41-48.

15. Secretaria de Salud. Reglamento de la Ley General de Salud en Materia de Investigación para la Salud. México: Diario Oficial de la Federación; 1982

16. Alonso C, Camacho M, Armendariz G, Alonso $C$, Ulloa $M$, Pérez $P$. Resiliencia y consumo de alcohol en adolescentes del municipio de Cunduacán, Tabasco. Cienc UANL. 2016;19(79):56-61.

17. Alonso-Castillo $M$, Yáñez-Loano $A$, Armendáriz García N. Funcionalidad familiar y consumo de alcohol en adolescentes de secundaria. Salud y drogas. 2017; 17(1):87-96.

18. Villa $R$, Castillo $D$, Velázquez $H$, Lomas G. Familia, tiempo libre y consumo de alcohol en estudiantes de preparatoria. Visión Edcativa IUNAES. 2020; 14(31):93-104.

19. Gámez-Medina M, Guzmán-Facundo $\mathrm{F}$, Ahumada-Cortez J, Alonso-Castillo M, Gheraldi-Donato E. Autoestima y consumo de alcohol en adolescentes escolarizados. Nure Investigación. 2017; 14(88):1-9.

20. Luna-Salas M, Villa-Rivas F, CastilloDíaz R, Velázquez-Hernández $\mathrm{N}$. Consumo de Alcohol y Agencia de Autocuidado en Adolescentes de una
Escuela Pública. Investigación de Enfermería en Adicciones y en el Cuidado de las Conductas de salud y Estilos de Vida Saludables. Editorial: La biblioteca; 2017.

21. Telumbre-Terrero J, Sánchez-Jaimes B. Consumo de alcohol en adolescentes del estado de Guerrero, México. Salud y drogas. 2015; 15(1)79-86.

22. Palomar J, Gómez N. Desarrollo de una escala de medición de la resiliencia con mexicanos (RESI-M). Interdisciplinaria. 2010; 27(1):7-22.

23. Gutiérrez M, Romero I. Resiliencia, bienestar subjetivo y actitudes de los adolescentes hacia el consumo de drogas en Angola. An psicol. 2014; 30(2):608-619.

24. Alvarez A, Zamora A, Hernández MA, Sánchez M, Barrón M, Mendoza MA. Resiliencia y consumo de alcohol y tabaco en estudiantes adolescentes de comunidades rurales. Anu investigación adicciones. 2011;16-19.

25. Hodder RK, Freund M, Bowman J, Wolfenden L, Gillham K, Dray J, et al. Association between adolescent tobacco, alcohol and illicit drug use and individual and environmental resilience protective factors. BMJ Open. 2016; 6(11):e012688. 
Financiamento: Os autores declaram que não houve financiamento.

Conflito de interesses: Os autores declaram não haver conflito de interesses.

Participação dos autores:

- Concepção: Villa-Rivas F, Castillo-Díaz R, Velázquez-Hernández N, Montelongo-Lugo JD.

- Desenvolvimento: Villa-Rivas F, Castillo-Díaz R, Velázquez-Hernández N, Montelongo-Lugo JD.

- Redação e revisão: Villa-Rivas F, Castillo-Díaz R, Velázquez-Hernández N, Montelongo-Lugo JD.

Como citar este artigo: Villa-Rivas F, Castillo-Díaz R, VelázquezHernández N, Montelongo-Lugo JD. Características resilientes y consumo de alcohol en adolescentes que viven en situación vulnerable. J Health NPEPS. 2021; 6(1):244-255.

Submissão: 23/11/2020

Aceito: 26/02/2021

Publicado: 01/06/2021 